\title{
RESEARCH
}

Open Access

\section{Multiparametric cardiovascular magnetic resonance characteristics and dynamic changes in myocardial and skeletal muscles in idiopathic inflammatory cardiomyopathy}

Yuanwei $\mathrm{Xu}^{1 \dagger}$, Jianhong Sun ${ }^{2 \dagger}$, Ke Wan ${ }^{3}$, Liuyu Yu ${ }^{4}$, Jie Wang ${ }^{1}$, Weihao $\mathrm{Li}^{1}$, Fuyuao Yang ${ }^{1}$, Jiayu Sun ${ }^{5}$, Wei Cheng ${ }^{5}$, David Mui', Qing Zhang ${ }^{1}$, Qibing Xie ${ }^{2^{*}}$ and Yucheng Chen ${ }^{1^{*}}$

\begin{abstract}
Background: Idiopathic inflammatory myopathy (IIM) manifest as systematic muscle involvement. Multiparametric cardiovascular magnetic resonance (CMR) could be a useful technique to detect systemic involvement and disease progression in IIM patients. This study aimed to describe the tissue characteristics and dynamic changes in myocardial and skeletal muscles after treatment in IIM patients.

Methods: Forty-four consecutively recruited IIM patients (49.0 \pm 12.0 years; 22 males) underwent 3 T CMR at first diagnosis, and 28 patients underwent follow-up scan after receiving standard treatment for more than 1 year. Thirty age- and sex-matched healthy subjects served as controls. The CMR protocol included: cines, T2-weighted (T2w), late gadolinium enhancement (LGE), T1 and T2 mapping, and extracellular volume (ECV) evaluated for the myocardium, and T1 and T2 mapping and ECV evaluated for skeletal muscles. Correlations between laboratory biomarkers and myocardial and skeletal tissue characteristics were analyzed. Comparisons between baseline and follow-up scans were performed using paired t-tests.

Results: At baseline, IIM patients showed significantly decreased hematocrit, higher left ventricular (LV) mass index, right ventricular (RV) volume index, myocardial and skeletal native T1, T2 mapping, and ECV than healthy controls. Significant correlations were found among myocardial native T1, T2 mapping, and ECV values and N-terminal pro btype natriuretic peptide (NT-proBNP) levels, and significant correlations between skeletal T2 mapping and inflammatory biomarkers in IIM patients.

During the follow-up, 28 patients underwent repeated CMR scan (median interval, 14.5 months, interquartile range: 13.2-15.5 months). Significant relief from clinical symptoms and decreased inflammatory biomarkers levels were observed. Significant reduction in myocardial native T1, T2, ECV, and skeletal native T1, T2, and ECV were observed (Continued on next page)
\end{abstract}

\footnotetext{
*Correspondence: xieqibing1971@163.com; chenyucheng2003@126.com

${ }^{\dagger}$ Yuanwei Xu and Jianhong Sun contributed equally to this work.

${ }^{2}$ Department of Rheumatology and Immunology, West China Hospital, Sichuan University, Chengdu, Sichuan 610041, People's Republic of China

'Department of Cardiology, West China Hospital, Sichuan University, Chengdu, Sichuan 610041, People's Republic of China

Full list of author information is available at the end of the article
}

C C The Author(s). 2020 Open Access This article is licensed under a Creative Commons Attribution 4.0 International License, which permits use, sharing, adaptation, distribution and reproduction in any medium or format, as long as you give appropriate credit to the original author(s) and the source, provide a link to the Creative Commons licence, and indicate if changes were made. The images or other third party material in this article are included in the article's Creative Commons licence, unless indicated otherwise in a credit line to the material. If material is not included in the article's Creative Commons licence and your intended use is not permitted by statutory regulation or exceeds the permitted use, you will need to obtain permission directly from the copyright holder. To view a copy of this licence, visit http://creativecommons.org/licenses/by/4.0/ The Creative Commons Public Domain Dedication waiver (http://creativecommons.org/publicdomain/zero/1.0/) applies to the data made available in this article, unless otherwise stated in a credit line to the data. 
(Continued from previous page)

during the follow-up assessment.

Conclusions: Both myocardial and skeletal muscles in newly diagnosed IIM patients show distinct characteristics on multiparametric CMR. In addition, significant changes were observed in patients showing clinical remission after effective treatment, which suggests that quantitative T1, T2, and ECV techniques may have potential clinical value in IIM patients.

Keywords: Idiopathic inflammatory myopathies, Cardiovascular magnetic resonance, Native T1 mapping, T2 mapping, Extracellular volume, Skeletal muscles

\section{Background}

Idiopathic inflammatory myopathies (IIM) are a heterogeneous group of autoimmune myositis affecting skeletal muscles and multiple internal organs [1]. The typical clinical manifestations of IIM include proximal muscle weakness, myalgia, and dyspnea related to respiratory muscle dysfunction due to systemic impairment of skeletal muscles. Myocardial involvement could be subclinical, but it is a major cause of adverse outcomes in patients with IIM [1-4]. Clinical manifestations of cardiac involvement in IIM patients can range from none to arrhythmia, heart failure, and even cardiac death [5]. Previous studies have found cardiac involvement manifesting as diastolic dysfunction or decreased longitudinal strain by echocardiography [6,7], abnormalities on electrocardiography (ECG) [6], presence of myocardial late gadolinium enhancement (LGE) [3, 8, 9], increased myocardial T1and T2 values [10-12], and increased extracellular volume (ECV) [13] by cardiovascular magnetic resonance (CMR). Although a few previous studies have reported the potential value of multiparametric CMR techniques in identifying cardiac involvement in patients with IIM $[10,11]$, there is still a lack of dynamic monitoring of myocardial histological changes during therapy. Meanwhile, skeletal muscle involvement is often the most severe and typical manifestation in patients with IIM. Previous studies have indicated the feasibility and potential value of evaluating thoracic skeletal tissue abnormality by using CMR $[10,12]$. However, the correlation between skeletal muscle inflammation measured by mapping techniques and disease activities related to biomarkers remains uncertain.

In the present study, we hypothesized that multiparametric CMR would help identify myocardial and skeletal involvement in patients with IIM and facilitate further dynamic monitoring of the effect of therapy during the clinical convalescence period of the disease.

\section{Methods}

\section{Study design and population}

This was a prospective observational cohort study involving 44 patients with newly diagnosed IIM who were consecutively enrolled from January 2017 to April 2018.
The diagnosis of IIM was made according to the criteria of the European Neuromuscular Centre (ENMC) International Workshop (2004) [14]. All patients were assessed by clinical symptoms, laboratory tests, electromyography (EMG) and muscle or skin biopsy. For patients with muscle weakness with or without rash, the elevated creatine kinase (CK) levels and abnormal EMG findings indicated muscle damage, and muscle biopsy was performed to confirm the diagnosis. IIM was classified as polymyositis (without rash), dermatomyositis (with rash), or autoimmune necrotizing myositis (without rash) according to specific muscular pathology. For patients without muscle weakness but rash, IIM was classified as amyopathic dermatomyositis if CK and EMG were normal but skin biopsy demonstrated reduced capillary density and deposition of the C5b-9 complement membrane attack complex on small blood-vessels along the dermal-epidermal junction. The exclusion criteria were as follows: (1) patients with other autoimmune or inflammatory diseases; (2) patients who had been diagnosed as having IIM and were treated with corticosteroids or immunosuppressive drugs; (3) patients with any known cardiovascular diseases, including coronary artery diseases, cardiomyopathy, valvular diseases, long-term poor controlled hypertension or other chronic cardiac conditions; (4) patients who were unable to tolerate CMR examination or had a contraindications to CMR; (5) patients with renal impairment defined by glomerular filtration rate $<30 \mathrm{ml} / \mathrm{min} / 1.73 \mathrm{~m}^{2}$; and (6) patients with incomplete or poor-quality images. For all patients with IIM, baseline image acquisition was performed before the administration of regular treatment. Thirty ageand sex-matched healthy controls without any known cardiac disease or hypertension, diabetes mellitus, cerebrovascular disease, nervous system disease, or pulmonary or renal diseases were included. The first CMR scan was performed within 1 week of the IIM diagnosis and the followup CMR scans were performed among patients who received at least 1 year of regular treatment.

All participants underwent the same CMR protocol and provided written informed consent. The study complied with the Declaration of Helsinki and ethical approval was obtained from the local institutional ethics committee at West China Hospital (2016355). 


\section{CMR examination}

All participants underwent CMR examinations on a $3 \mathrm{~T}$ scanner (MAGNETOM, Trio a Tim system; Siemens Healthineers, Erlangen, Germany) using an eightchannel phased-array body coil. All the images were acquired by breath-holding and ECG gating. A balanced steady-state free precession (bSSFP) sequence was used for cine images with continuous sampling from the basal to the apical levels on short-axis views and 2-, 3-, 4chamber long-axis views. The typical scan parameters were as follows: repetition time (TR), $34 \mathrm{~ms}$; echo time (TE), $1.3 \mathrm{~ms}$; flip angle, $50^{\circ}$; field of view (FOV), $280 \times$ $340 \mathrm{~mm}^{2}$; matrix size, $162 \times 192$; average temporal resolution, 35-45 ms; and slice thickness, $8 \mathrm{~mm}$ with no gap. Cardiac T2-weighted (T2w) images were acquired with the short TI inversion recovery (STIR) sequence on three short-axis views (basal, mid-ventricular and apical) and the 4-chamber long-axis view. The typical scan parameters were as follows: $\mathrm{TR}=2 \mathrm{RR}$ intervals during breath-hold; TE, $67 \mathrm{~ms}$; flip angle, $180^{\circ}$; FOV, $280 \times 340$ $\mathrm{mm}^{2}$; matrix size, $166 \times 256$; and slice thickness, $10 \mathrm{~mm}$. Phase-sensitive inversion recovery (PSIR) sequence with a segmented FLASH readout scheme was performed 10-15 min after gadolinium (Magnevist; Bayer Schering Pharma, Berlin, Germany) injection with $0.15 \mathrm{mmol} / \mathrm{kg}$ per bolus on consecutive short-axis views from the basal to apical level of LV and 2-, 3-, and 4-chamber long-axis views to obtain the LGE images. The typical scan parameters were as follows: TR, $700 \mathrm{~ms}$; TE, $2.0 \mathrm{~ms}$; delay time after the inversion pulse, $300-380 \mathrm{~ms}$; flip angle, $20^{\circ}$; FOV, $260 \times 340 \mathrm{~mm}^{2}$; matrix size, $116 \times 192$; and slice thickness, $8 \mathrm{~mm}$. T1 mapping images were acquired on three short-axis and 4-chamber views, as in the previous sequence, by using the Modified Look-Locker Inversionrecovery (MOLLI) sequence with a $5 \mathrm{~b}(3 \mathrm{~b}) 3 \mathrm{~b}$ (b stands for heartbeat) scheme and a $4 b(1 b) 3 b(1 b) 2 b$ scheme before and $10-15 \mathrm{~min}$ after the gadolinium injection. The typical scan parameters were as follows: TR, $300 \mathrm{~ms}$; TE, $1.2 \mathrm{~ms}$; flip angle, $35^{\circ}$; FOV, $270 \times 320 \mathrm{~mm}^{2}$; matrix size, $144 \times 256$; and slice thickness, $8 \mathrm{~mm}$. T2 mapping images were acquired by $\mathrm{T}_{2}$-prepared single-shot bSSFP technique before administration of gadolinium on the short- and long-axis views identical to those associated with $\mathrm{T} 1$ mapping images. The typical scan parameters were as follows: TR, $240 \mathrm{~ms}$; TE, $1.0 \mathrm{~ms}$; flip angle, $12^{\circ}$; FOV, $320 \times 340 \mathrm{~mm}^{2}$; matrix, $114 \times 176$; slice thickness, $8 \mathrm{~mm}$; and T2 preparation pulse with $0,30,55 \mathrm{~ms}$ spin echo times.

\section{CMR imaging analysis}

\section{Function and volume assessments}

Biventricular volumes, ejection fraction, and LV mass were analyzed using a commercially dedicated postprocessing software (Qmass, version 8.2, Medis Medical
Imaging Systems, Leiden, The Netherlands) according to the standardized protocol of Society of Cardiovascular Magnetic Resonance (SCMR) post-processing guideline [15]. Biventricular volume and LV mass were indexed to the body surface area (BSA).

\section{T2-weighted ratio assessment}

The T2w signal intensity (SI) ratio was calculated as the ratio of the signal of the LV myocardium on midventricular short-axis $\left(\mathrm{SI}_{\text {myo }}\right)$ to the signal of the adjacent skeletal muscles $\left(\mathrm{SI}_{\mathrm{sk}}\right)$ on the same slice.

\section{$T 1$ and $T 2$ mapping assessments}

Native T1, T2 relaxation times, and ECV of both the myocardium and skeletal muscles were measured on the same mid-ventricular short-axis slice by using Medis Suite 2.1 (Medis). The myocardial region of interest (ROI) was manually delineated on the end-diastolic phase with the endo- and epicardial contours to avoid endocardial trabeculations, blood pool, and epicardial fat. The selection of ROI on T1 and T2 mapping images of the adjacent skeletal muscles included five groups (pectoralis major, subscapularis, infraspinatus, upper arm, and erector spinae muscles) with carefully avoidance of adipose tissue. The analyses of tissue characteristics of skeletal muscles were performed by calculating the average values for the five groups. The native $\mathrm{T} 1$ and T2 relaxation times of both myocardium and skeletal muscles were generated from motion-corrected images by fitting an exponential decay curve of each pixel in ROIs. For both myocardial and skeletal muscles, the T1 relaxation time before and after contrast injection was used to calculate ECV according to the following formula: (1-hematocrit) $\left(1 / \mathrm{T} 1_{\text {muscle post }}-1 / \mathrm{T} 1_{\text {muscle pre }}\right) /$ $\left(1 / \mathrm{T} 1_{\text {blood post }}-1 / \mathrm{T} 1_{\text {blood pre }}\right)$. Hematocrit was obtained on the same day as the CMR examination in normal controls and within 3-days of CMR in patients. Care was taken to exclude image artifacts on myocardiaum and skeletal muscles. The illustrative images of bSSFP cine, native T1 mapping, T2 mapping, and ECV on short-axis views of a healthy subjects and one IIM patient at baseline (visit 1) and CMR follow-up (visit 2) are shown in Fig. 1.

\section{LGE assessment}

The presence of LGE was judged by two independent experienced CMR observers blinded to patient information. The extent of LGE was measured with the threshold defined as 3 standard derivations (SDs) above the signal of the remote normal myocardial region [15].

\section{Clinical data, treatment, and follow-up}

Clinical presentation and symptoms including symmetric proximal muscle weakness, rash, myalgia, and dysphagia, 


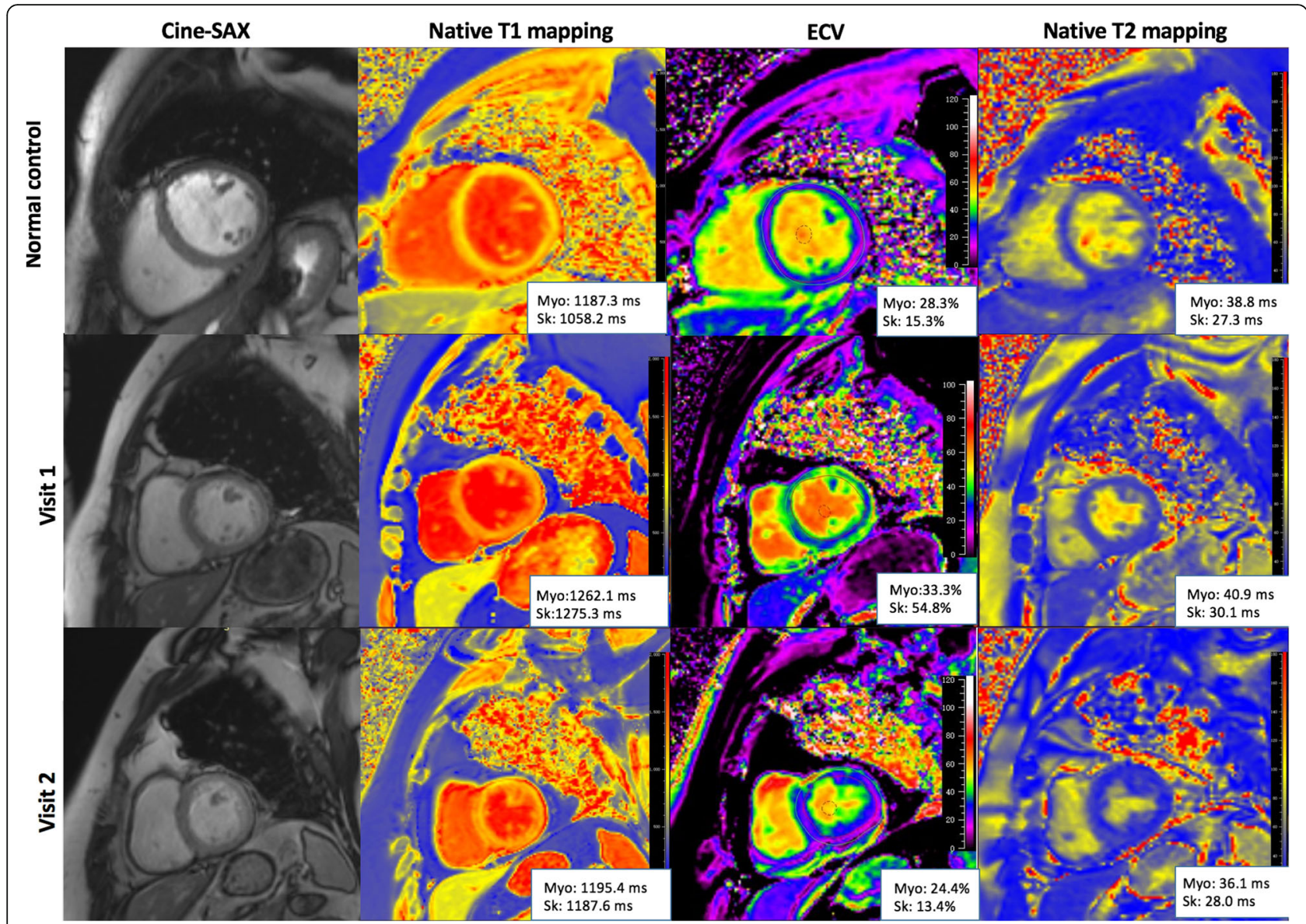

Fig. 1 Illustrative balanced steady state free precession (bSSFP) cine, native T1, T2, and extracellular volume fraction (ECV) images on left ventricular short-axis views. Images of one healthy subject (top) and one idiopathic inflammatory myopathy (IIM) patient at baseline (visit 1) and CMR follow-up (visit 2). Abbreviation: Myo = myocardial, Ske = skeletal, SAX = short-axis; ECV = extracellular volume

and laboratory biomarkers including hematocrit (Hct), C-reactive protein (CRP), erythrocyte sedimentation rate $(\mathrm{ESR})$, creatine kinase (CK), creatine kinase-myocardial band (CKMB), cardiac troponins $\mathrm{T}$ (cTnT), and NTproBNP were evaluated both at the time of the first diagnosis and at the follow-up within 3 days of the CMR examination in IIM patients.

After the diagnosis of IIM, corticosteroids were chosen as the first-line medications in all patients, and immunosuppressive therapies including cyclophosphamide, methotrexate, and/or mycophenolate mofetil were personalized based on the patient's disease severity and immune response. High-dose prednisone was initially administrated with gradually tapered doses until the lowest dose which could keep the patient in sustained remission [16].

The follow-up data were last updated in Jun 2019 by collecting information by outpatient follow-up and telephone contact. Clinical remission of IIM was defined as (1) the improvement of muscle strength to normal level, accompanied by being able to move with resisting force;
(2) remission of dermatitis, including the extinction of heliotrope, periorbital oedema, Gottron's papules/sign, V-sign, shawl sign, and holster sign [14]; and (3) the normalization of laboratory tests results, including CK, ESR, and CRP levels [16].

\section{Statistical analysis}

The normality of variables was tested using the Kolmogorov-Smirnov method. Continuous variables were expressed as mean $\pm \mathrm{SD}$ or median (interquartile range) values. Categorical variables were expressed as percentages. Parameters between IIM patients and healthy controls were compared with independentsample T, Mann-Whitney or chi-square tests as appropriate. The differences between baseline and follow-up parameters in the subgroup of IIM patients with followup CMR scans were assessed by paired t-test or MannWhitney U test. Logarithmic transformation was performed on biomarker parameters for normal distribution. Correlations between CMR parameters and biomarkers were evaluated using Pearson's correlation 
(r). Reproducibility of tissue mapping parameters was evaluated in 20 randomly selected IIM patients. The intra- and inter-observer variability was assessed by Bland-Altman analysis (bias and 95\% limit of agreement), coefficients of variation (CoVs), and intra-class correlation coefficients (ICCs). Two-tailed $p<0.05$ was considered statistically significant. Statistical analyses were performed using MedCalc (version 11.5.1.0, Mariakerke, Belgium).

\section{Results}

\section{Patient characteristics at baseline}

We enrolled 46 IIM patients, among which two patients (4.3\%) were excluded due to poor image quality. The study cohort consisted of 44 newly diagnosed IIM patients, and the demographic, clinical, and CMR parameters of IIM patients and healthy controls are summarized in Table 1. At baseline, IIM patients showed a significantly lower Hct, higher LV mass index and right ventricular (RV) diastolic volume index, and lower RV ejection fraction (RVEF) in comparison with age- and sex-matched controls. In the analysis of myocardial tissue characteristics, newly diagnosed IIM patients showed significantly longer native T1 (1275 \pm 82 vs. $1199 \pm 47 \mathrm{~ms}, p<0.001)$ and T2 $(44.2 \pm 4.0$ vs. $38.3 \pm$ $2.9 \mathrm{~ms}, p<0.001)$ relaxation times, and higher ECV $(32.3 \% \pm 6.2 \%$ vs. $26.6 \% \pm 2.4 \%, p<0.001)$ than normal controls. Eleven patients (25\%) were LGE-positive with an average extent of $12.0 \% \pm 3.1 \%$ by the threshold of 3SD. For the evaluation of skeletal tissue characteristics, IIM patients showed significantly longer native T1 $(1287 \pm 1499$ vs. $1107 \pm 70 \mathrm{~ms}, p<0.001)$ and $\mathrm{T} 2(34.4 \pm$ 3.1 vs. $20.6 \pm 1.4 \mathrm{~ms}, p<0.001)$ relaxation times, and higher ECV $(31.1 \% \pm 12.8 \%$ vs. $13.6 \% \pm 3.1 \%, p<0.001)$ at baseline compared to normal controls.

\section{Correlation between tissue characteristics and cardiac structure, function and biomarkers at baseline}

The increased myocardial native T1, T2, and ECV values were significantly correlated with a larger LV volume index, higher LV mass index, and lower LV ejection fraction (EF) Table 2. Meanwhile, elongation of the myocardial T2 relaxation time was significantly correlated with a decrease in RVEF. The myocardial native $\mathrm{T} 1(\mathrm{r}=0.547$, $p<0.001)$, T2 $(\mathrm{r}=0.406, p=0.005)$, and ECV $(\mathrm{r}=0.313$, $p=0.037$ ) values showed moderate positive correlation with the $\log$ NT-proBNP. Among the multiparametric tissue characteristics of skeletal muscles, we found that skeletal T2 relaxation times showed significant positive correlation with the $\log \operatorname{ESR}(r=0.430, p=0.004), \log$ CK-MB $(\mathrm{r}=0.471, p=0.001)$, and $\log \mathrm{cTnT}(\mathrm{r}=0.563$, $p<0.001)$ levels, while skeletal native T1 and ECV did not show significant correlations with the levels of laboratory biomarkers. The results are presented in Fig.2.
In the study, we also found that elevation of myocardial T2 value was significantly correlated with the skeletal $\mathrm{T} 2$ value $(\mathrm{r}=0.317, p=0.036)$, while no significant correlation was found among T1 or ECV values on myocardium with those on skeletal muscles. The scatterplots are presented in Fig. 3.

\section{Subgroup analysis of patients with follow-up CMR scans}

During the follow-up, 28 IIM patients were arranged to consecutively undergo repeated scans with a median CMR interval of 14.5 mo (interquartile: $13.2-15.5 \mathrm{mo}$ ). Those who have not been treated regularly $(n=2)$, had a treatment time of less than 1 year $(n=6)$, or were reluctant to undergo a second CMR for personal reasons were excluded from the subgroup analysis. All 28 patients reached clinical remission during the CMR followup, with no patients demonstrating proximal muscle weakness or skin rash. Patients also showed significantly decreased CRP, CK, CK-MB, TnT and NT-proBNP levels and increased Hct during follow-up in comparision with those at baseline. While a significant decrease in LV mass index was observed (baseline: $51.2 \pm 12$. vs. follow-up: $45.2 \pm 10.2 \mathrm{~g} / \mathrm{m}^{2}, \quad p=0.011$ ), no significant changes were observed in the biventricular volume index or ejection function. Seven LGE-positive patients at baseline were still LGE-positive at CMR follow-up with a trend of a reduced extent of LGE with no statistically significant difference (baseline: $11.8 \pm 2.9 \%$ vs. follow-up: $8.1 \pm 2.0 \%, p=\mathrm{NS})$. Patients showed significantly decreased myocardial T1 mapping (baseline: $1289 \pm 53$ vs. follow-up: $1262 \pm 59 \mathrm{~ms}, p=0.013$ ), T2 mapping (baseline: $44.4 \pm 4.0 \mathrm{~ms}$ vs. follow-up: $41.8 \pm 4.2 \mathrm{~ms}, p=0.001$ ), and ECV (baseline: $30.7 \pm 4.1 \%$ vs. follow-up: $27.6 \pm$ $4.4 \%, \mathrm{p}=0.001$ ) during the follow-up CMR. However, the myocardial native T1 and T2 values were still significantly higher than those for normal controls. The comparison between baseline and follow-up CMR of the subgroup IIM patients is summarized in Table 3 and Fig. 4.

In the assessment of skeletal tissue characteristics, patients showed significantly decreased skeletal native T1 relaxation time (baseline: $1268 \pm 103$ vs. baseline: $1154 \pm$ $73 \mathrm{~ms}, p<0.001$ ), T2 relaxation time (baseline: $35.5 \pm 3.5$ follow-up: $29.7 \pm 2.8 \mathrm{~ms}, p<0.001)$ time, and ECV (baseline: $29.2 \% \pm 11.1 \%$ vs. baseline: $21.9 \% \pm 11.2 \%, p=$ 0.011), with the native T1, T2 and ECV values of skeletal muscles still significantly higher than those of normal controls. The results are summarized in Table 4 and presented in Fig. 4.

\section{Inter- and inter-observer reproducibility}

Table 4 shows the inter- and intra-reproducibility of the measurements obtained with native T1 mapping, T2 mapping, and ECV for myocardium and skeletal 
Table 1 Baseline characteristics of IIM patients compared with those of normal controls

\begin{tabular}{|c|c|c|c|}
\hline Characteristics (units) & IIM patients $(n=44)$ & Healthy Controls $(n=30)$ & $P$ value \\
\hline Age (years) & $49.0 \pm 12.0$ & $48.2 \pm 15.6$ & 0.232 \\
\hline Males, $n(\%)$ & $22(50.0 \%)$ & 15 (50.0\%) & 1.000 \\
\hline BMI $\left(\mathrm{kg} / \mathrm{m}^{2}\right)$ & $22.4 \pm 3.0$ & $22.8 \pm 2.8$ & 0.465 \\
\hline Systolic blood pressure, mmHg & $122 \pm 14$ & $121 \pm 11$ & 0.673 \\
\hline Diastolic blood pressure, $\mathrm{mmHg}$ & $78 \pm 8$ & $72 \pm 5$ & 0.512 \\
\hline Heart rate & $76.5 \pm 9.7$ & $74.1 \pm 7.2$ & 0.592 \\
\hline Disease duration (years) & $0.5(0.2,2.0)$ & - & - \\
\hline \multicolumn{4}{|l|}{ Clinical presentation } \\
\hline Proximal muscle weakness, n (\%) & $41(93.2 \%)$ & - & - \\
\hline Rash, $n(\%)$ & $29(65.9 \%)$ & - & - \\
\hline Myalgia, $n(\%)$ & $23(52.3 \%)$ & - & - \\
\hline Dysphagia, $n(\%)$ & $17(38.6 \%)$ & - & - \\
\hline \multicolumn{4}{|l|}{ Comorbidity } \\
\hline Hypertension, n (\%) & $6(13.6 \%)$ & - & - \\
\hline Diabetes, $n(\%)$ & $1(2.3 \%)$ & - & - \\
\hline \multicolumn{4}{|l|}{ Biomarkers } \\
\hline Hct & $0.40 \pm 0.05$ & $0.43 \pm 0.03$ & 0.003 \\
\hline CRP (mg/L) & $4.3(1.7-9.7)$ & - & - \\
\hline ESR $(\mathrm{mm} / \mathrm{h})$ & $28(23-47)$ & - & - \\
\hline CK (U/L) & $316(79-2202)$ & - & - \\
\hline CK-MB (ng/mL) & $20.6(5.3-54.0)$ & - & - \\
\hline $\mathrm{CTnT}(\mathrm{g} / \mathrm{L})$ & $96(40-269)$ & - & - \\
\hline NT-proBNP (pg/mL) & $226(85-747)$ & - & - \\
\hline \multicolumn{4}{|l|}{ Medication } \\
\hline Steroids, $n(\%)$ & $44(100 \%)$ & - & - \\
\hline Methotrexate, $n(\%)$ & $22(50.0 \%)$ & - & - \\
\hline Cyclophosphamide, $n$ (\%) & $12(27.3 \%)$ & - & - \\
\hline Chloroquine, $n(\%)$ & $18(40.9 \%)$ & - & - \\
\hline Azathioprine, $n(\%)$ & $4(9.1 \%)$ & - & - \\
\hline \multicolumn{4}{|l|}{ Cardiac structure and function } \\
\hline LVEDVi, $\mathrm{mL} / \mathrm{m}^{2}$ & $82.7 \pm 26.6$ & $77.1 \pm 13.3$ & 0.300 \\
\hline LVESVi, $\mathrm{mL} / \mathrm{m}^{2}$ & $33.9 \pm 25.1$ & $29.1 \pm 8.1$ & 0.428 \\
\hline LVEF (\%) & $61.4 \pm 11.8$ & $62.0 \pm 5.2$ & 0.958 \\
\hline LVmassi $\left(\mathrm{g} / \mathrm{m}^{2}\right)$ & $50.8 \pm 15.0$ & $43.6 \pm 8.2$ & 0.037 \\
\hline RVEDVi, $\mathrm{mL} / \mathrm{m}^{2}$ & $80.9 \pm 22.2$ & $68.7 \pm 18.5$ & 0.042 \\
\hline RVESVi, $\mathrm{mL} / \mathrm{m}^{2}$ & $38.2 \pm 17.1$ & $34.3 \pm 10.5$ & 0.146 \\
\hline RVEF (\%) & $53.6 \pm 9.8$ & $57.2 \pm 5.2$ & 0.055 \\
\hline \multicolumn{4}{|l|}{ Myocardial tissue characterization } \\
\hline T2w ratio & $1.3 \pm 0.4$ & $1.2 \pm 0.4$ & 0.259 \\
\hline LGE, $n(\%)$ & $11(25 \%)$ & 0 & - \\
\hline LGE extent, \% & $12.0 \pm 3.1$ & 0 & - \\
\hline Native T1 mapping-myocardial, ms & $1275 \pm 82$ & $1199 \pm 47$ & $<0.001$ \\
\hline T2 mapping-myocardial, ms & $44.2 \pm 4.0$ & $38.3 \pm 2.9$ & $<0.001$ \\
\hline ECV-myocardial, \% & $32.3 \pm 6.2$ & $26.6 \pm 2.4$ & $<0.001$ \\
\hline
\end{tabular}


Table 1 Baseline characteristics of IIM patients compared with those of normal controls (Continued)

\begin{tabular}{llll}
\hline Characteristics (units) & IIM patients $(n=44)$ & Healthy Controls $(n=30)$ & $P$ value \\
\hline Skeletal tissue characterization & & & $<0.001$ \\
Native T1 mapping-skeletal, ms & $1287 \pm 149$ & $1107 \pm 70$ & $<\mathbf{0 . 0 0 1}$ \\
T2 mapping-skeletal, ms & $34.4 \pm 3.1$ & $26.0 \pm 1.4$ & $<\mathbf{0 . 0 0 1}$ \\
ECV-skeletal, \% & $31.1 \pm 12.8$ & $13.6 \pm 3.1$ & $<$ \\
\hline
\end{tabular}

Abbreviations: $B M I$ body mass index, SBP systolic blood pressure, DBP diastolic blood pressure, $H R$ heart rate, NYHA New York Heart Association functional classification, Hct hematocrit, CRP C-reactive protein, ESR erythrocyte sedimentation rate, CK creatine kinase, CK-MB creatine kinase-MB, TnT Troponins T, NT-proBNP $\mathrm{N}$-terminal pro b-type natriuretic peptide, LVEDVi left ventricular end-diastolic volume index, LVESVi left ventricular end-systolic volume index, LVmassi left ventricular mass index, LVEF left ventricular ejection fraction, RVEDVi right ventricular end-diastolic volume index, RVESVi right ventricular end-systolic volume index, RVEF right ventricular ejection fraction, $T 2 W$ T2-weighted ratio, $L G E$ late gadolinium enhancement, ECV extracellular volume fraction

Values in bold indicate $P$ values $<0.05$

$P$ values for comparison between IIM patients and normal controls

muscles. We found the reproducibility of skeletal mapping parameters (CoV ranging from 2.12 to $4.43 \%)$ to be slightly worse than those of myocardial mapping parameters ( $\mathrm{CoV}$ ranging from 0.86 to $2.19 \%$ ).

\section{Discussion}

This study demonstrates the value of multiparametric CMR in detecting myocardial and skeletal muscles involvement in IIM patients, and dynamic changes after effective treatment. The main findings of the study are as follows: (1) multiparametric CMR could detect both myocardial and skeletal involvement in newly diagnosed IIM patients in comparision with normal controls. (2) In newly diagnosed IIM patients, the myocardial T1, T2, and ECV were correlated with LV volume, function, and the log NT-proBNP level, while skeletal T2 was significantly correlated with biomarkers reflecting disease activity. (3) The follow-up CMR showed significantly decreased myocardial T1, T2, and ECV, as well as skeletal T1, T2, and ECV, which may indicate that the involvement of myocardium and skeletal muscles was reversible. This is the first study to show the potential role of multiparametric CMR in non-invasively monitoring the treatment effect on both cardiac and skeletal systems of IIM patients.

\section{Myocardial and skeletal muscles involvement in IIM}

It is not uncommon for the myocardium to be affected in systemic inflammatory diseases. In this context, the clinical value of CMR parametric mapping techniques in quantifying myocardial tissue alterations has been gaining recognition in many diseases [17]. Ntusi et al. demonstrated that T1 mapping could provide added value as a biomarker for diffuse myocardial fibrosis in rheumatoid arthritis patients [18]. Biesbroek et al. found that ECV and T1 mapping were significantly associated with the disease activity in ankylosing spondylitis patients and may be a potential marker for disease monitoring [19]. In the present study, newly diagnosed IIM patients showed significantly elevated T1, T2, and ECV values in comparison with normal controls, and these findings are consistent with recent CMR studies of IIM patients [10, 12]. We also found that the myocardial native $\mathrm{T} 1, \mathrm{~T} 2$ and ECV values were significantly correlated with the NT-proBNP level, which may suggest that.

quantitative mapping parameters can be used as potential imaging markers for detecting subclinical cardiac involvement in newly diagnosed IIM patients. The significant correlation between myocardial ECV and serum CRP level, as well as the significant correlation between myocardial and skeletal T2 values, may indicate that the degree of myocardial inflammation is positively correlated with the systematic inflammation level. In addition, for the assessment of skeletal tissue characteristics, elevated skeletal T2 relaxation time was shown to be correlated with biomarkers such as the ESR, CK, CK-MB, and cTnT levels. Our findings suggested that the skeletal inflammation reflected by

Table 2 Correlation between myocardial tissue characteristics with biventricular volume, ejection function, and LV mass at baseline

\begin{tabular}{|c|c|c|c|c|c|}
\hline & LVEDVi & LVmassi & LVEF (\%) & RVEDVi & RVEF (\%) \\
\hline \multirow[t]{2}{*}{ native $T 1, \mathrm{~ms}$} & $r=0.392$ & $r=0.358$ & $r=-0.506$ & $r=0.116$ & $r=-0.264$ \\
\hline & $p=0.044$ & $p=0.027$ & $p=0.001$ & $p=0.490$ & $p=0.110$ \\
\hline \multirow[t]{2}{*}{$\mathrm{T} 2 \mathrm{~ms}$} & $r=0.383$ & $r=0.377$ & $r=-0.374$ & $r=0.278$ & $r=-0.340$ \\
\hline & $p=0.018$ & $p=0.022$ & $p=0.021$ & $p=0.090$ & $p=0.037$ \\
\hline \multirow[t]{2}{*}{$\mathrm{ECV}, \%$} & $r=0.310$ & $r=0.329$ & $r=-0.521$ & $r=0.044$ & $r=-0.206$ \\
\hline & $p=0.030$ & $p=0.034$ & $p=0.001$ & $p=0.794$ & $p=0.215$ \\
\hline
\end{tabular}

Abbreviations: LVEDVi left ventricular end-diastolic volume index, LVmassi left ventricular mass index, LVEF left ventricular ejection fraction, RVEDVi right ventricular end-diastolic volume index, RVEF right ventricular ejection fraction

Values in bold indicates $P$ values $<0.05$ 

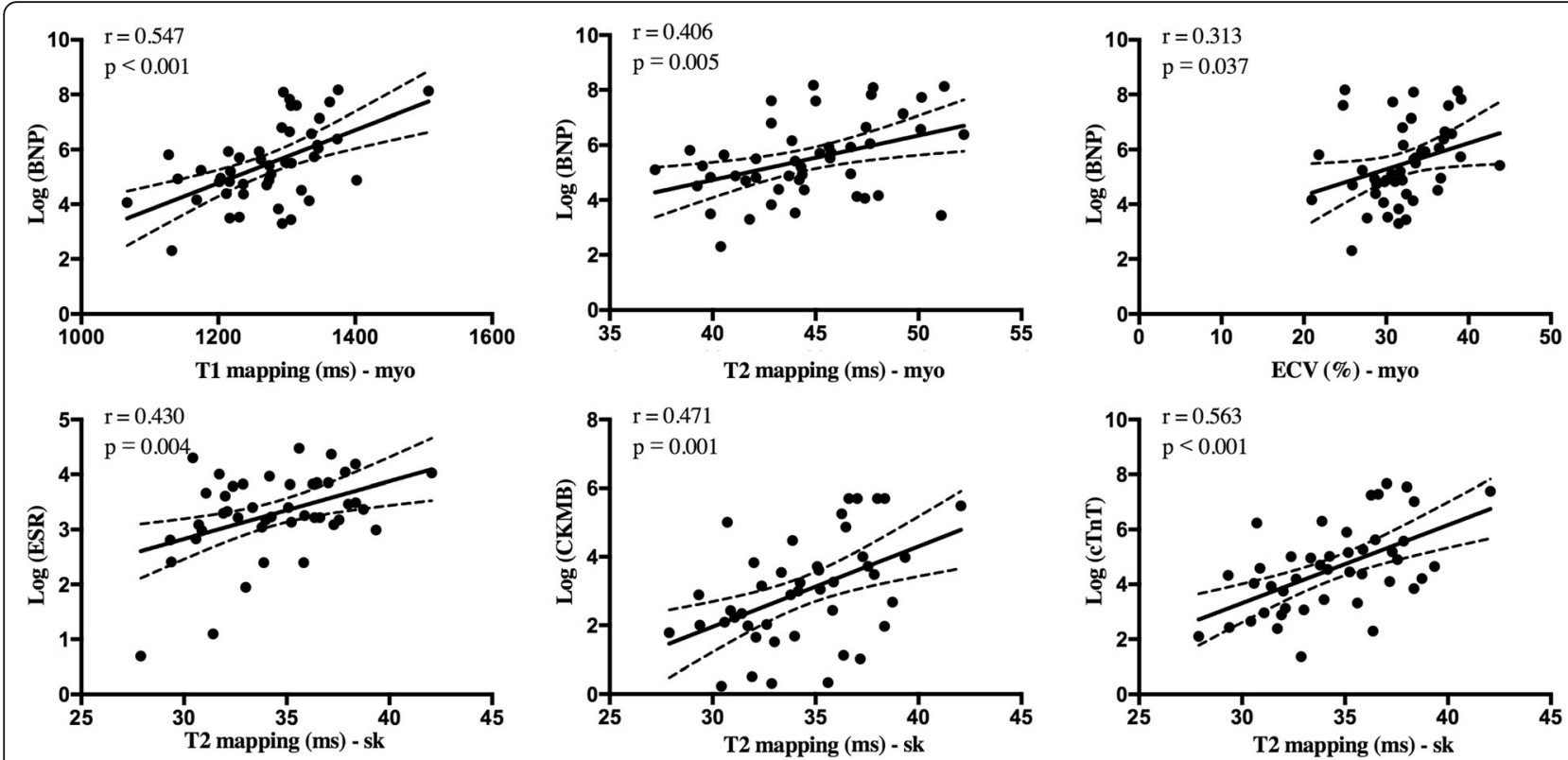

Fig. 2 Correlation between biomarkers and quantitative myocardial and skeletal tissue characteristics at baseline

CMR multiparametric mapping techniques is related to the disease activity.

\section{Dynamic changes in myocardial and skeletal tissue characteristics}

CMR mapping technologies have shown good reproducibility and sensitivity in dynamic monitoring for different diseases. Hinojar et al. found that lupus myocarditis patients had elevated myocardial native $\mathrm{T} 1$ and $\mathrm{T} 2$ relaxation times and showed a significant decrease after intensive therapy, which suggests an attenuation of myocardial inflammation [20]. Bohnen et al. found that native myocardial T1 and T2 measurements provide excellent performance for monitoring the healing of myocarditis with a significant decline based on the stage of acute myocarditis [21]. Spieker et al. also found T2 mapping to be a good tool for monitoring myocardial inflammation in patients with suspected acute myocarditis [22]. Alkhalil et al. demonstrated that the anatomical area at risk may best be quantified by $\mathrm{T} 1$ mapping undertaken in the first week after reperfusion [23]. However, some previous studies have presented discrepant findings regarding the dynamic changes associated with cardiac involvement in IIM patients. Allanore et al. studied four patients and found markedly reduced LGE areas and decreased hypokinesia after 6 months of corticosteroid and immunosuppressive therapy [24]. In contrast, Mavrogeni et al. found that the signs of myocarditis were still positive on CMR reevaluation after 3 months of steroid treatment with clinical remission [25]. Péter et al. reevaluated IIM patients 3 months after starting treatment by using echocardiography and found a significant improvement in LV and RV systolic function, despite also observing deterioration of diastolic dysfunction [25]. The uncertain conclusions in previous studies may be attributable to differences in patient selection, approaches used to evaluate cardiac abnormalities, different follow-up intervals, and the insufficient sample sizes.
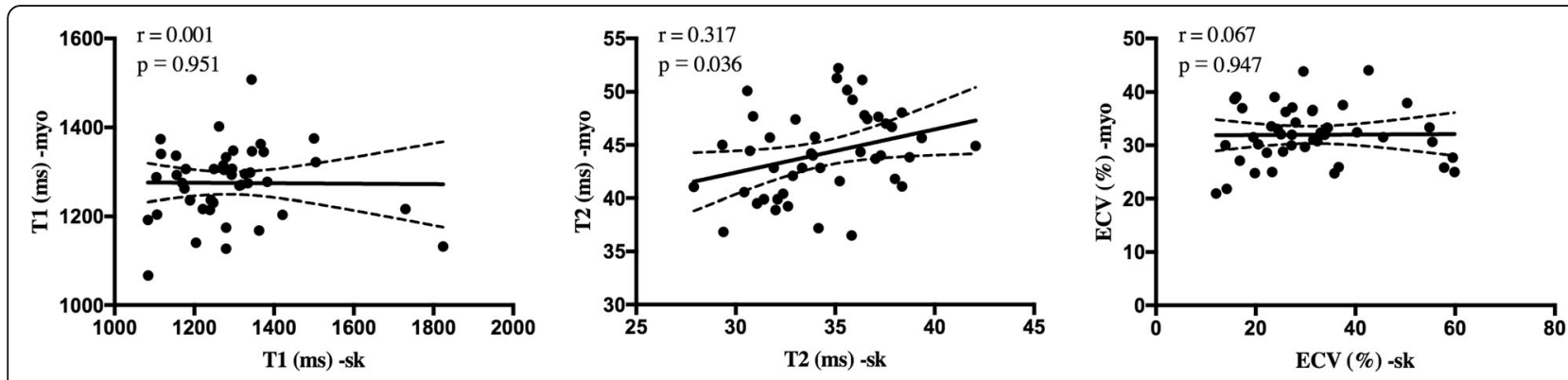

Fig. 3 Correlation between myocardial tissue mapping characteristics and skeletal muscles at baseline 
Table 3 Clinical biomarkers and CMR parameters at baseline and follow-up among IIM patients with CMR follow-up

\begin{tabular}{|c|c|c|c|}
\hline & Baseline $(n=28)$ & Follow-up $(n=28)$ & $P^{*}$ value \\
\hline \multicolumn{4}{|l|}{ Biomarker } \\
\hline Hct & $0.40 \pm 0.05$ & $0.43 \pm 0.04$ & 0.005 \\
\hline CRP (mg/L) & $5.7(2.8-17.9)$ & $2.2(1.8-4.1)$ & 0.024 \\
\hline $\operatorname{ESR}(\mathrm{mm} / \mathrm{h})$ & $26(23-47)$ & $22(5-33)$ & 0.064 \\
\hline CK (U/L) & $479(83-1060)$ & $106(75-148)$ & 0.038 \\
\hline CK-MB (ng/mL) & $24.7(7.1-74.6)$ & $2.5(1.0-4.8)$ & $<0.001$ \\
\hline $\mathrm{CTnT}(\mathrm{g} / \mathrm{L})$ & $102(60-480)$ & $17(10-67)$ & $<0.001$ \\
\hline BNP $(p g / m L)$ & $235(72-1184)$ & $50(26-109)$ & 0.007 \\
\hline \multicolumn{4}{|l|}{ Structural and function } \\
\hline LVEDVi, $\mathrm{m}^{3} / \mathrm{m}^{2}$ & $83.7 \pm 19.4$ & $82.3 \pm 18.0$ & 0.703 \\
\hline LVESVi, $\mathrm{ml}^{3} / \mathrm{m}^{2}$ & $34.0 \pm 15.3$ & $34.3 \pm 13.8$ & 0.549 \\
\hline LVmassi, $\mathrm{g} / \mathrm{m}^{2}$ & $51.2 \pm 12.5$ & $45.2 \pm 10.2$ & 0.011 \\
\hline LVEF, \% & $59.4 \pm 8.7$ & $60.5 \pm 10.5$ & 0.395 \\
\hline RVEDVi, $\mathrm{ml}^{3} / \mathrm{m}^{2}$ & $81.8 \pm 16.2$ & $79.8 \pm 11.6$ & 0.279 \\
\hline RVESVi, $\mathrm{ml}^{3} / \mathrm{m}^{2}$ & $39.3 \pm 11.8$ & $37.4 \pm 8.0$ & 0.073 \\
\hline RVEF, \% & $53.2 \pm 6.2$ & $55.2 \pm 6.8$ & 0.492 \\
\hline \multicolumn{4}{|c|}{ Myocardial tissue characteristics } \\
\hline T2w ratio & $1.3 \pm 0.5$ & $1.3 \pm 0.4$ & 0.621 \\
\hline LGE presence, n(\%) & $7(25 \%)$ & $7(25 \%)$ & 1 \\
\hline LGE extent, \% & $11.8 \pm 2.9$ & $8.1 \pm 2.0$ & 0.239 \\
\hline Native T1-myo, ms & $1289 \pm 53$ & $1262 \pm 59$ & 0.013 \\
\hline T2 mapping-myo, ms & $44.4 \pm 4.0$ & $41.8 \pm 4.2$ & 0.001 \\
\hline ECV-myo, \% & $30.7 \pm 4.1$ & $27.6 \pm 4.4$ & 0.001 \\
\hline \multicolumn{4}{|c|}{ Skeletal tissue characteristics } \\
\hline Native T1-sk, ms & $1268 \pm 103$ & $1154 \pm 73$ & $<0.001$ \\
\hline T2 mapping-sk, ms & $34.5 \pm 3.5$ & $29.7 \pm 2.8$ & $<0.001$ \\
\hline ECV-sk, \% & $29.2 \pm 11.1$ & $21.9 \pm 11.2$ & 0.011 \\
\hline
\end{tabular}

Abbreviations: Hct hematocrit, CRP C-reactive protein, ESR erythrocyte sedimentation rate, $C K$ creatine kinase, $C K-M B$ creatine kinase-MB, $T n T$ troponin T, NT-proBNP N-terminal pro b-type natriuretic peptide, LVEDVi left ventricular end-diastolic volume index, LVESVi left ventricular end-systolic volume index, LVmassi left ventricular mass index, LVEF left ventricular ejection fraction, $R V E D V i$ right ventricular end-diastolic volume index, RVESVi right ventricular end-systolic volume index, RVEF right ventricular ejection fraction, T2W T2weighted ratio, $L G E$ late gadolinium enhancement, $E C V$ extracellular volume fraction

Values in bold indicate $P$ values $<0.05$

$P^{*}$ value for comparison between baseline and follow-up characteristics among IIM patients

In the present study, we found that newly diagnosed IIM patients had significantly elevated T1, T2, and ECV values on both myocardium and skeletal muscles, which are consistent with the findings of previous studies [10, 12]. In addition, we also demonstrated for the first time that multiparametric CMR can detect dynamic changes in both myocardial and skeletal tissue characteristics after effective treatment.

Although the cellular and molecular pathophysiology underlying the myocardial involvement in IIM patients is not clear, the increased T2 relaxation time at baseline suggested that myocardial inflammation might be a major pathophysiological change in IIM patients [26]. Consistent with this speculation, we found a significant decrease in the myocardial T2 relaxation time at followup, which paralleled the remission of clinical symptoms and improvements in serum inflammatory biomarkers. In addition, the significant reduction in the myocardial mass index could be partially explained by the remission of myocardial inflammation. Skeletal T2 also showed a significant decrease under clinical remission. This finding suggests that IIM patients have both myocardial and skeletal inflammation at the newly diagnosed period, and that acute inflammation and edema are reversible after effective treatment. Both myocardial and skeletal T2 values served as feasible and sensitive markers for detecting myocardial and skeletal inflammation and monitoring the therapeutic effects in IIM patients. Although we found that the clinical application of these changes may be limited due to their small absolute change values, the study still provides clues for the potential application of individualized dynamic monitoring of the effect of treatment.

We found that IIM patients had elevated T1 values at both newly diagnosed and clinical remission stages in comparison with normal controls. While a previous study found that native T1 shows high diagnostic accuracy in discriminating the acute and convalescent stages of myocarditis [27], the inconsistent results of the current study indicate that the myocardial involvement in IIM patients may not only include acute inflammation but also diffuse fibrosis. The histological studies had demonstrated that the native $\mathrm{T} 1$ mapping relaxation time is affected by diffuse fibrosis [28, 29] and myocardial inflammation [30]. Whether the myocardial diffuse fibrosis in IIM patients is permanent or reversible over a longer term is not clear, and observational studies with a longer follow-up period are needed.

One previous study had demonstrated that in cases with coexisting inflammation and diffuse fibrosis, the ECV would reflect the sum of both pathologies [31]. ECV has shown excellent discriminative value in differentiating myocarditis patients from healthy subjects [21, $32]$, and declined significantly with the recovery of myocarditis [21]. In our study, myocardial ECV provided good discriminative value between newly diagnosed IIM patients and normal controls, with a significant decrease in the ECV of both the myocardium and skeletal muscle at follow-up CMR. Thus, ECV is also a promising tissue characterization marker for detecting subtle abnormalities and monitoring the recovery from inflammation of myocardial and skeletal tissue.

In contrast to the findings for quantitative $\mathrm{T} 1, \mathrm{~T} 2$, and ECV techniques, the T2W images showed limited ability 

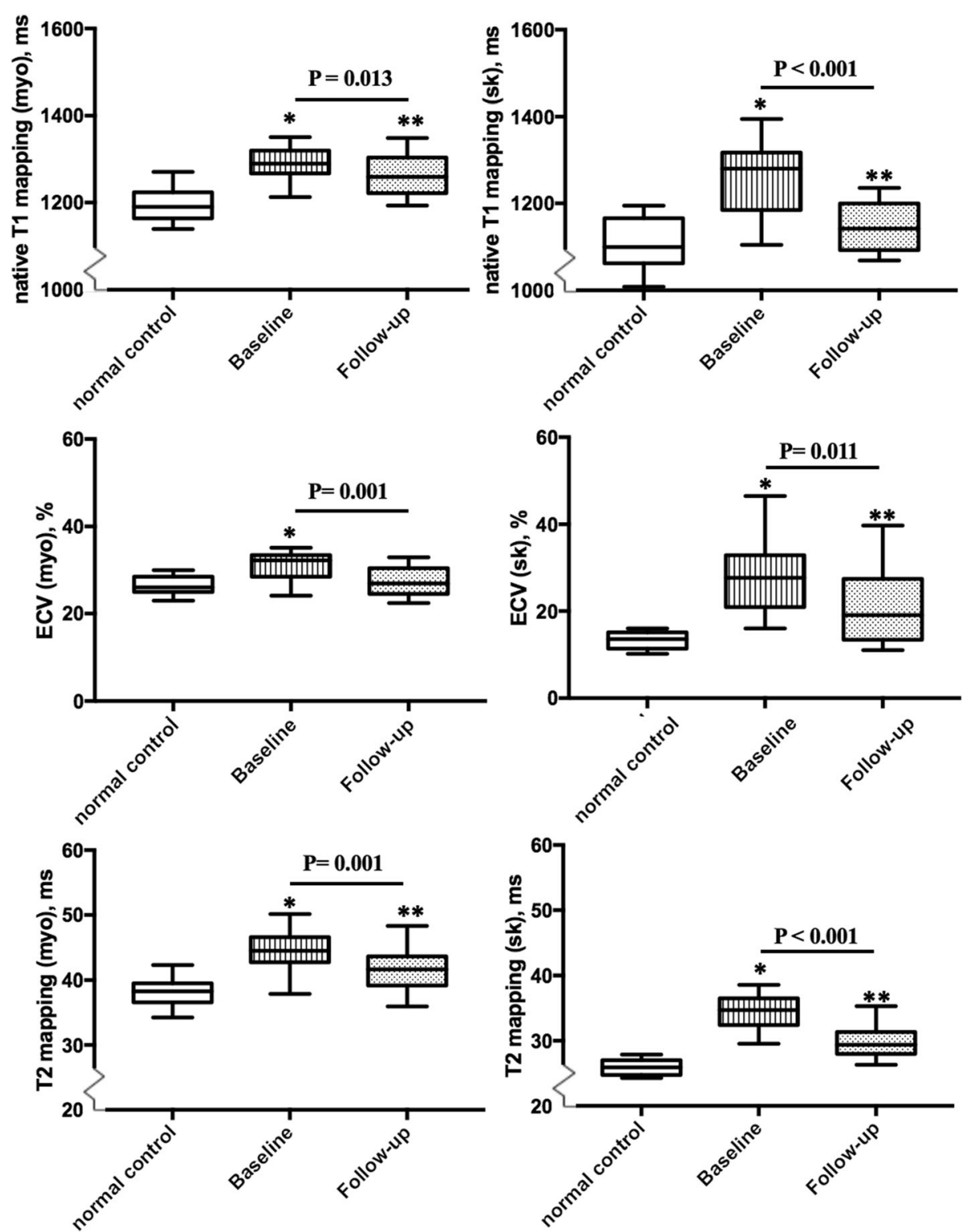

Fig. 4 Comparison of myocardial and skeletal tissue characteristics [a (myocardial), b (skeletal): native T1 mapping; c (myocardial), d (skeletal): T2 mapping; e (myocardial), $\mathbf{f}$ (skeletal): ECV] among healthy controls and the IIM subgroup patients $(n=28)$ at baseline and follow-upAbbreviations: myo = myocardium; sk = skeletal muscle.The lower and upper limits of the box represent the 25th and 75th percentiles and whiskers represent the 10th to 90th range.The baseline and follow-up boxplots of IIM patients composed of 28 patients who had CMR follow-up.

to distinguish IIM patients from normal controls. This lack of sensitivity may be explained by the concurrent abnormality on skeletal muscles [33]. The T2 mapping technique offered the advantages of quantitative measurement of $\mathrm{T} 2$ relaxation times without the need for skeletal muscles as reference. Our finding suggests that T2W images have limited value for evaluation of IIM patients, consistent with the findings of a previous study [26].

In the current study, IIM patients with positive LGE at baseline still showed LGE during follow-up, without a significant decrease in the LGE extent. This may be explained by the fact that LGE reflects irreversible myocardial injury [34]. Previous studies have indicated that the presence of LGE may reflect chronic and active myocardial inflammation [34, 35].

\section{Correlations between tissue characterization parameters} and cardiac function and structure

In the current study, we found that elevated myocardial native T1, T2, and ECV significantly correlated with LV systolic dysfunction and enlargement, while the myocardial T2 value also correlated with RV dysfunction. These results suggest that myocardial inflammation and the 
Table 4 Intra- and inter-observer reproducibility of the measurements of myocardial and skeletal mapping characteristics in IIM patients

\begin{tabular}{lllll}
\hline & Bias & $95 \%$ LOA & CoV (\%) & ICC \\
\hline Intra-observer & & & & \\
Native T1-myo (ms) & -4.7 & $(-34.5-25.1)$ & 0.86 & 0.99 \\
T2 mapping-myo (ms) & 0.3 & $(-1.3-1.8)$ & 1.28 & 0.99 \\
ECV-myo (\%) & -0.4 & $(-2.2-1.3)$ & 2.19 & 0.99 \\
Native T1-sk (ms) & -9.0 & $(-83.5-65.5)$ & 2.12 & 0.98 \\
T2 mapping-sk (ms) & -0.2 & $(-2.6-2.1)$ & 2.81 & 0.96 \\
ECV-sk (\%) & -0.1 & $(-2.7-2.6)$ & 2.71 & 0.99 \\
Inter-observer & & & & \\
Native T1-myo (ms) & -5.1 & $(-39.2-29.0)$ & 0.98 & 0.98 \\
T2 mapping-myo (ms) & 0.3 & $(-1.5-2.1)$ & 1.51 & 0.98 \\
ECV-myo (\%) & -0.2 & $(-1.8-1.3)$ & 1.80 & 0.98 \\
Native T1-sk (ms) & -10.8 & $(-126.6-118.3)$ & 3.52 & 0.93 \\
T2 mapping-sk (ms) & -0.2 & $(-3.8-3.2)$ & 4.43 & 0.91 \\
ECV-sk (\%) & 0.3 & $(-2.7-3.4)$ & 3.17 & 0.99 \\
\hline
\end{tabular}

Abbreviation: LOA limit of agreement, CoV coefficient of variation, ICC intraclass correlation coefficients, $\mathrm{Cl}$ confidence intervals, $E C V$ extracellular volume fraction

possible presence of myocardial fibrosis in IIM patients can adversely impact cardiac function and structure.

\section{Study limitations}

This study had several limitations. First, this is a singlecenter study with a relatively small number of patients. Further studies with larger sample sizes and external validation are needed. Second, not all IIM patients enrolled at baseline underwent follow-up CMR scans. Although this could potentially lead to study bias, we focused on the subsequent observation CMR scans to analyze the dynamic changes in both myocardial and skeletal tissue characterizations. Third, the imaging data for the myocardium were not supported by biopsy findings. Future studies on the prognostic value of multiparametric CMR in IIM patients are required to validate these findings.

\section{Conclusions}

The study demonstrates that multiparametric CMR could allow early detection of myocardial and skeletal muscles involvement in IIM patients, and help monitor dynamic changes after effective treatment.

\section{Abbreviations}

BSA: Body surface area; bSSFP: Balanced steady-state free precession; CK: Creatine kinase; CK-MB: Creatine kinase-myocardial band; CMR: Cardiovascular magnetic resonance; CoV: Coefficients of variation; CRP: C-reaction protein; CTnT: Cardiac troponins T; ECG Electrocardiogram ECV: Extracellular volume fraction; EMG: Electromyogram; ENMC: European Neuromuscular Centre; ESR: Erythrocyte sedimentation rate; FOV: Field of view; HCT: Hematocrit; HF: Heart failure; ICC: Intra-class correlation coefficient; IIM: Idiopathic inflammatory myopathies; LGE: Late gadolinium enhancement; LV: Left ventricle/left ventricular; LVEF: Left ventricular ejection fraction; MOLLI: Modified Look-Locker inversion recovery; NT-proBNP: N-terminal pro b-type natriuretic peptide; PSIR: Phase sensitive inversion recovery; ROI: Region of interest; RV: Right ventricle/right ventricular; RVEF: Right ventricular ejection fraction; SCMR: Society of Cardiovascular Magnetic Resonance; SD: Standard derivation; SI: Signal intensity; $\mathrm{SI}_{\text {myo }}$ : Signal intensity of myocardium; $\mathrm{SI}_{\text {sk: }}$ Signal intensity of skeletal muscle; STIR: Short-tau inversion recovery; T2w: T2-wighted; TE: Echo time; TR: Repetition time

\section{Acknowledgements}

Not applicable.

\section{Authors' contributions}

YCC initiated the study, defined its design, headed its coordination and reviewed the manuscript. QIX and QZ headed the coordination and reviewed the manuscript. YWX designed the study, acquired the image and clinical data, read the images, performed statistical analysis and drafted the manuscript. JHS made substantial contributions to study design, patients' enrollment, clinical data acquisition, data interpretation and manuscript writing. KW and LYY made contributions to the study design, the analysis and interpretation of the data. JW, WHL and FYY contributed to the image and clinical data acquisition, JYS and WC acquired the image data. DM revised language of the manuscript. All author read and approved the final manuscript.

\section{Funding}

This work was supported by the National Natural Science Foundation of China (Grant No. 81571638) and 1.3.5 project for disciplines of excellenceClinical Research Incubation Project, West China Hospital, Sichuan University (ZYJC18013).

\section{Availability of data and materials}

The datasets acquired and/or analyzed during the current study are available from the corresponding author on reasonable request.

\section{Ethics approval and consent to participate}

The study protocol complies with the Declaration of Helsinki and was approved by ethics committee of West China Hospital, Sichuan University (2016355). Written informed consent was obtained from all participants.

\section{Consent for publication}

Not applicable.

\section{Competing interests}

Not applicable.

\section{Author details}

${ }^{1}$ Department of Cardiology, West China Hospital, Sichuan University, Chengdu, Sichuan 610041, People's Republic of China. ${ }^{2}$ Department of Rheumatology and Immunology, West China Hospital, Sichuan University, Chengdu, Sichuan 610041, People's Republic of China. ${ }^{3}$ Department of Geriatrics, West China Hospital, Sichuan University, Chengdu, Sichuan Province, China. ${ }^{4}$ Department of Cardiology, Union Hospital, Tongji Medical College, Huazhong University of Science and Technology, Wuhan, Wuhan province, China. ${ }^{5}$ Department of Radiology, West China Hospital, Sichuan University, Chengdu, Sichuan Province, China. ${ }^{6}$ Department of Medicine, Cardiovascular Division, University of Pennsylvania, Philadelphia, USA.

Received: 13 August 2019 Accepted: 16 March 2020

Published online: 09 April 2020

\section{References}

1. Lundberg IE. The heart in dermatomyositis and polymyositis. Rheumatology. 2006;45(Suppl 4):iv18-21

2. Schwartz T, Diederichsen LP, Lundberg IE, Sjaastad I, Sanner H. Cardiac involvement in adult and juvenile idiopathic inflammatory myopathies. RMD Open. 2016;2(2):e000291.

3. Sramko M, Franeková J, Kautznerová D. Utility of combination of cardiac magnetic resonance imaging and high-sensitivity cardiac troponin $T$ assay 
in diagnosis of inflammatory cardiomyopathy. Am J Cardiol. 2013;111(2): 258-64.

4. Gupta R, Wayangankar SA, Targoff IN, Hennebry TA. Clinical cardiac involvement in idiopathic in fl ammatory myopathies : a systematic review. Int J Cardiol. 2011;148:261-70.

5. Diederichsen LP. Cardiovascular involvement in myositis. 2017:29:598-603.

6. Diederichsen LP, Simonsen JA, Diederichsen AC, Hvidsten S, Hougaard M, Junker $\mathrm{P}$, et al. Cardiac abnormalities in adult patients with polymyositis or dermatomyositis as assessed by noninvasive modalities. Arthritis Care Res. 2016;68:1012-20

7. Zhong Y, Bai W, Xie Q, Sun J, Tang H, Rao L. Cardiac function in patients with polymyositis or dermatomyositis : a three-dimensional speckle-tracking echocardiography study. Int J Cardiovasc Imaging. 2018;34:683-93.

8. Rosenbohm A, Buckert D, Gerischer N, Ludolph AC, Bernhardt P. Early diagnosis of cardiac involvement in idiopathic inflammatory myopathy by cardiac magnetic resonance tomography. J Neurol. 2015;262:949-56.

9. Mavrogeni S, Douskou M, Manoussakis MN. Contrast-enhanced CMR imaging reveals myocardial involvement in idiopathic inflammatory myopathy without cardiac manifestations. JACC Cardiovasc Imaging. 2011;4: $1324-5$

10. Huber AT, Lamy J, Bravetti M, Bouazizi K, Bacoyannis T, Roux C. Comparison of MR T1 and T2 mapping parameters to characterize myocardial and skeletal muscle involvement in systemic idiopathic inflammatory myopathy (IIM ). Eur Radiology. 2019;29:5139-47.

11. Yu L, Sun J, Sun J, Li J, Dong Y, Zhou X, et al. Early detection of myocardial involvement by $\mathrm{T} 1$ mapping of cardiac MRI in idiopathic inflammatory myopathy. J Magn Reson Imaging. 2018:48:415-22.

12. Huber AT, Bravetti M, Lamy J, Bacoyannis T, Roux C, De Cesare A, et al. Noninvasive differentiation of idiopathic inflammatory myopathy with cardiac involvement from acute viral myocarditis using cardiovascular magnetic resonance imaging T1 and T2 mapping. J Cardiovasc Magn Reson. 2018;20: 11.

13. Bravetti M, Kachenoura N, Roux C, Chabi ML, Rigolet A, Benveniste O, et al. Detection of subclinical cardiac involvement in inflammatory myopathy by CMR T1 relaxometry. J Cardiovasc Magn Reson. 2016;18(Suppl 1):P254.

14. Hoogendijk JE, Amato AA, Lecky BR, Choy EH, Lundberg E, Rose MR, et al. 119th ENMC international workshop: Trial design in adult idiopathic inflammatory myopathies, with the exception of inclusion body myositis, 10-12 October 2003, Naarden, the Netherlands. Neuromuscul Disord. 2004; 14:337-45.

15. Fogel MA, Friedrich MG, Kim RJ, Knobelsdorff-brenkenhoff F. Von. Standardized image interpretation and post processing in cardiovascular magnetic resonance : Society for Cardiovascular Magnetic Resonance ( SCMR ) Board of Trustees Task Force on Standardized Post Processing. J Cardiovasc Magn Reson. 2013;15:35.

16. Ernste FC, Reed AM. Idiopathic inflammatory myopathies: current trends in pathogenesis, clinical features, and up-to-date treatment recommendations. Mayo Clin Proc. 2013;88:83-105.

17. DeLong ER, DeLong DM, Clarke-Pearson DL. Comparing the areas under two or more correlated receiver operating characteristic curves: a nonparametric approach. Biometrics. 1988;44:837.

18. Messroghli DR, Moon JC, Ferreira VM, Grosse-Wortmann L, He T, Kellman P, et al. Clinical recommendations for cardiovascular magnetic resonance mapping of $\mathrm{T} 1, \mathrm{~T}_{2}, \mathrm{~T}^{*}$ and extracellular volume: a consensus statement by the Society for Cardiovascular Magnetic Resonance (SCMR) endorsed by the European Association for Cardiovascular Imaging (EACVI). J Cardiovasc Magn Reson. 2017;19:75.

19. Ntusi NA, Piechnik SK, Francis JM, et al. Diffuse myocardial fibrosis and inflammation in rheumatoid arthritis: insights from CMR T1 mapping. JACC Cardiovasc Imaging. 2015;8:526-36.

20. Hinojar R, Foote L, Sangle S, Marber M, Mayr M, Carr-white G, et al. Native $\mathrm{T} 1$ and $\mathrm{T} 2$ mapping by CMR in lupus myocarditis : disease recognition and response to treatment. Int J Cardiol. 2016;222:717-26.

21. Bohnen S, Radunski UK, Lund GK, Ojeda F, Looft Y, Senel M, et al. Tissue characterization by $\mathrm{T} 1$ and $\mathrm{T} 2$ mapping cardiovascular magnetic resonance imaging to monitor myocardial inflammation in healing myocarditis. Eur Hear J Cardiovasc Imaging. 2017;18:744-51.

22. Spieker M, Haberkorn S, Gastl M, Behm P, Katsianos S, Horn P, et al. Abnormal T2 mapping cardiovascular magnetic resonance correlates with adverse clinical outcome in patients with suspected acute myocarditis. J Cardiovasc Magn Reson. 2017:1-9.
23. Alkhalil M, Borlotti A, De Maria GL, Gaughran L, Langrish J, Lucking A, et al. Dynamic changes in injured myocardium, very early after acute myocardial infarction, quantified using T1 mapping cardiovascular magnetic resonance. J Cardiovasc Magn Reson. 2018;20:1-10.

24. Allanore $Y, L A$ OV. Effects of corticosteroids and immunosuppressors on idiopathic inflammatory myopathy related myocarditis evaluated by magnetic resonance imaging. Ann Rheum Dis. 2006;65:249-52.

25. Mavrogeni S, Bratis K, Karabela G, Stavropoulos E, Sfendouraki E, Kolovou G. Myocarditis during acute inflammatory myopathies evaluation using clinical criteria and cardiac magnetic resonance imaging. Int J Cardiol. 2013;164:3-4.

26. Thavendiranathan P, Walls M, Giri S, Verhaert D, Moore S, Simonetti OP, et al. Improved detection of myocardial involvement in acute inflammatory cardiomyopathies using T2 mapping. Circ Cardiovasc Imaging. 2012;5:102-10.

27. Hinojar R, Foote L, Bs C, Ucar EA, Jackson T, Bs C, et al. Native T1 in discrimination of acute and convalescent stages in patients with clinical diagnosis of myocarditis. JACC Cardiovasc Imaging. 2015;8:37-46.

28. Lee S-p, Lee W, Lee JM. Assessment of diffuse myocardial fibrosis by using MR imaging in asymptomatic. Radiology. 2015;274:359-69.

29. Bull S, White SK, Piechnik SK, Flett AS, Ferreira VM, Loudon M, et al. Human non-contrast $\mathrm{T} 1$ values and correlation with histology in diffuse fi brosis. 2013;932-7.

30. Bull S, White SK, Piechnik SK, Flett AS, Ferreira VM, Loudon M, et al. Human non-contrast $\mathrm{T} 1$ values and correlation with histology in diffuse fibrosis. Heart. 2013;99:932-7.

31. Lurz JA, Luecke C, Lang D, Besler C, Rommel K, Klingel K, et al. CMR derived extracellular volume fraction as a marker for myocardial fibrosis: the importance of coexisting myocardial inflammation. JACC Cardiovasc Imaging. 2018;11:38-45.

32. Bohnen S, Adam G, Blankenberg S, Muellerleile K, Page SEE. CMR in patients with severe myocarditis: diagnostic value of quantitative tissue markers including extracellular volume imaging. JACC Cardiovasc Imaging. 2014;7: $667-75$.

33. Jaworski C, Looi J, lles LM, Mclean CA, Taylor AJ, Hare JL. Bright muscle, qeak heart, Bad Start? Hear Lung Circ. 2014;23:293-4.

34. Friedrich MG, Sechtem U, Schulz-menger J, Alakija P, Cooper LT, White JA, et al. Cardiovascular magnetic resonance in myocarditis: a JACC White paper. J Am Coll Cardiol. 2010;53:1475-87.

35. Nordin S, Kozor R, Bulluck H, Castelletti S, Rosmini S, Abdel-Gadir A, et al. Cardiac Fabry disease with late gadolinium enhancement is a chronic inflammatory cardiomyopathy. J Am Coll Cardiol. 2016;68:1707-8.

\section{Publisher's Note}

Springer Nature remains neutral with regard to jurisdictional claims in published maps and institutional affiliations.

Ready to submit your research? Choose BMC and benefit from:

- fast, convenient online submission

- thorough peer review by experienced researchers in your field

- rapid publication on acceptance

- support for research data, including large and complex data types

- gold Open Access which fosters wider collaboration and increased citations

- maximum visibility for your research: over $100 \mathrm{M}$ website views per year

At BMC, research is always in progress.

Learn more biomedcentral.com/submissions 